\title{
Research on the Hub City of "OBOR" Based on the Fuzzy Comprehensive Evaluation Approach
}

\author{
Min Xie ${ }^{1, a}$, Chunhua $\mathrm{Li}^{2, \mathrm{~b}}$ \\ ${ }^{1}$ Jiangxi Science \&Technology Normal University, Nanchang 330013, P.R. China \\ ${ }^{2}$ School of Science, East China Jiaotong University, Nanchang 330013, China \\ aminxie7612781@163.com, bchunhuali66@163.com
}

\begin{abstract}
Keywords: fuzzy sets ; comprehensive evaluation; OBOR; GDP; urbanization(industrialization) rate; investment enviroment.

Abstract. As a fuzzy mathematics method, fuzzy comprehensive evaluation approach is used in various fields, and achieves some good results. The purpose of this paper is to use this approach to research overall conditions of the hub city of "OBOR"--Nanchang. After obtaining the relevant data, it is concluded that the present overall conditions of the city of Nanchang is good by using the fuzzy mathematics comprehensive evaluation approach.
\end{abstract}

\section{Introduction}

On March 28, 2015, the national top-level design construction plan of "One Belt One Road (for short, OBOR)" was finally appeared. Authorized by the state council, on the same day, the national development and reform commission, the ministry of foreign affairs, the ministry of commerce jointly issued the "push to build the silk road economic belt and the 21st century the vision and action of the Marine silk road", officially released "OBOR" of the ultimate empire. In the design of this plan, Nanchang of jiangxi province and Chengdu, Wuhan, Changsha, Zhengzhou, Hefei and other cities, are listed as "OBOR" important node in the city. Nanchang is the strategy of "OBOR" important node in the city, as the national strategy of "OBOR", will bring new opportunities to nanchang urbanization development. In the future, Nanchang will rely on the connection between the north and the south of the unique geographical advantage, unique industrial advantages, and the chance of "OBOR" all the way, to promote economic and social development.

Lotfi Zadeh[1] introduced the notion of a fuzzy subset of a set in 1965 . His seminal paper has opened up new insights and applications in a wide range of scientific fields. It offers new tools and a new approach to model the imprecision and uncertainty present in phenomena that do not have sharp boundaries. Since then, a series of research on fuzzy sets has come out expounding the importance of the concept and its applications to logic, algebra theory etc( see, [2-6] ). In order to learn about overall conditions of the hub city of "OBOR" —-Nanchang, in this paper, we shall use fuzzy mathematics comprehensive evaluation approach to evaluate Nanchang from GDP(gross domestic product), urbanization rate, industrialization rate and investment enviroment, respectively.

\section{Preliminaries.}

In this section, we first state some known results and notations.

Let $X$ be a non-empty set. A fuzzy subset $A$ of $X$ is a function of $X$ to the unit interval $[0,1]$. For all $x \in X, A(x)$ can be thought of as the degree of membership of $x$ in $A$.

Definition II.1 ${ }^{[6]}$ Let $X$ be a non-empty set, and let $A$ be a fuzzy subset of $X, t \in[0,1]$, $A_{t}=\{x \in X \mid A(x) \geq t\}$. Then $A_{t}$ is called a $t-$ cut.

Definition II. $2^{[6]}$ Let $A, B$ be two fuzzy subsets of a semigroup $S$. For all $x \in S$, we give the following definitions:

(1) $A \supseteq B \Leftrightarrow A(x) \geq B(x) ;(2)(A \cap B)(x)=A(x) \wedge B(x),(A \cup B)(x)=A(x) \vee B(x)$;

(3) $(A \circ B)(x)=\left\{\begin{array}{cc}\vee_{x=y z}\{A(y) \wedge B(z)\} & \text { if } \exists y, z \in S, x=y z \\ 0 & \text { otherwise }\end{array}\right.$. 
Definition II. $3^{[6]}$ Let $X$ be a non-empty set. A function $u$ from $X \times X$ into the unit interval [ 0,1$]$ is called $a$ fuzzy relation on $X$. Let $u, v$ be two fuzzy relations on $X$. Then the product $u \circ v$ of $u$ and $v$ is defined by $u \circ v(a, b)=\vee_{x \in S}\{u(a, x) \wedge v(x, b)\}$.

Definition II.4 ${ }^{[7]}$ A matrix $R=\left(r_{i j}\right)_{m \times n}$ is called a $m \times n$ fuzzy matrix, if $r_{i j} \in[0,1]$ for all $i=1,2, \cdots, m$ and $j=1,2, \cdots, n$.

Obviously, if the set we study is finite, then fuzzy relation can be turned into fuzzy matrix.

Next, we shall give the definition of a fuzzy mathematics comprehensive evaluation approach. Evaluation is to appraise and judge the pros and cons of things according to the given conditions; Composite refers to the conditions of judgment contain multiple factors or more indicators. Comprehensive evaluation is to make a comprehensive judgment on the object affected by multiple factors. At the present, although there are quite many comprehensive evaluation methods, only two kinds of them are usually used. The following statements are the definition of them.

Definition II. $5^{[7]} \quad$ Evaluation of total score method is to decide the merits of the scheme by listing the evaluation items according to the evaluation object, determining the evaluation grade of the each project, and expressing as a fraction.

Definition II.6 ${ }^{[7]}$ Weighted scoring method mainly consider the factors or the index in the evaluation of the status and roles which are not the same and can not be equal to the factors or the index, introduce the concept of weight, and embody the factors or the index in the evaluation of different position or different function.

Obviously, the weighted scoring method is more reasonable than the evaluation of total score method. A fuzzy mathematics comprehensive evaluation approach belongs to a special case of the weighted scoring method. So, the fuzzy comprehensive evaluation decision is also called fuzzy comprehensive decision or fuzzy multivariate decision. The mathematical model of fuzzy comprehensive evaluation model can be divided into one-level model and multilevel model. In this paper, we shall use one-level model, which is introduced as follows:

Let $U=\left\{x_{1}, x_{2}, \cdots, x_{n}\right\}$ be $n$ kinds of factors, $V=\left\{v_{1}, v_{2}, \cdots, v_{m}\right\} m$ kinds of evaluations. Because of various factors of the position, function is also different, and weight is also different. The $n$ kinds of judgments are not absolute yes or no. So comprehensive evaluation should be a fuzzy subset on $V: \underline{B}=\left(b_{1}, b_{2}, \cdots, b_{m}\right) \in F(V)$, where $F(V)$ denotes the set of all fuzzy subsets of $V$, and $b_{j}$ reflects the position for the $j$ kind of evaluation $v_{j}$. That is, $\underline{B}\left(v_{j}\right)=b_{j}$. The evaluation method and procedures are as follows:

(i) the factor set $U=\left\{x_{1}, x_{2}, \cdots, x_{n}\right\}$, where $x_{i}(i=1,2, \cdots, n)$ is influential to the things the $i$ th element.

(ii) the evaluation set $V=\left\{v_{1}, v_{2}, \cdots, v_{m}\right\}$, where $v_{j}(j=1,2, \cdots, m)$ is the $j$ th level of the evaluation.

(iii) the single factor evaluation method: determine each factor of the degree of membership of each evaluation class, and get fuzzy matrix

$$
R=\left(R_{1}, R_{2}, \cdots, R_{n}\right)^{T}=\left(r_{i j}\right)_{n \times m}=\left(\begin{array}{ccc}
r_{11} & \cdots & r_{1 m} \\
\vdots & \ddots & \vdots \\
r_{n 1} & \cdots & r_{n m}
\end{array}\right),
$$

where the $i$ th line $R_{i}$ in $R$ reflects the degree of membership of the $i$ th factor of the evaluated object for evaluation of various grades, and column $j$ reflects that the various factors of evaluation object choose respectively from the $j$ grade level of the evaluation set. $(U, V, R)$ forms a comprehensive decision-making model. 
(iv) determine the weight of each factor $\underline{A}=\left(a_{1}, a_{2}, \cdots, a_{n}\right) \in F(U)$, and $\sum_{i=1}^{n} a_{i}=1,0 \leq a_{i} \leq 1$, where $a_{i}$ is the weight of the $i$ th factor.

(v) comprehensive evaluation. $\underline{B}=\underline{A} \circ R=\left(b_{1}, b_{2}, \cdots, b_{m}\right)$ is for the comprehensive evaluation of things.

\section{An Example of Fuzzy Comprehensive Evaluation about the Overall Conditions of Nanchang High-tech Zone.}

This paper selects Nanchang high-tech zone for comprehensive evaluation. Nanchang high-tech zone, there are more than 2000 enterprises cluster, including the Microsoft, the United States Merck, Germany's bertelsmann, Japan Hitachi, Switzerland ABB, nearly 20 Fortune Global 500 and the United States kohler, Germany G\&D, Taiwan east yuan, umc, envada, such as multinational corporations, to form the biological medicine, electronic information and application software, precision machinery manufacturing machine integrates light pillar industry, new material four characteristics. In recent years, the main economic indicators in the zone at an annual rate of more than $30 \%$ per head is growing rapidly. Its economy has been among the top of the provincial development zone. Along with the advancement of "OBOR", high-tech as the most development potential of the plate, nanchang of jiangxi province and even the national appeal will present the growth of the blowout, high-tech zone is important development opportunities.

We shall establish mathematical model according to the above described method.

(i) the factor set $U=\left\{x_{1}, x_{2}, x_{3}, x_{4}\right\}$, where $x_{1}, x_{2}, x_{3}$ and $x_{4}$ denote GDP(gross domestic product), urbanization rate, industrialization rate and investment enviroment, respectively.

(ii) the evaluation set $V=\left\{v_{1}, v_{2}, v_{3}, v_{4}\right\}$, where $v_{1}, v_{2}, v_{3}$ and $v_{4}$ denote very good, good, not so

good and not good, respectively.

(iii) the single factor evaluation. Here are 100 people's data to various factors. These results are listed in the following Table 1:

\section{Table 1.}

\begin{tabular}{|l|lccc|}
\hline \multirow{2}{*}{ the factor set $U$} & \multicolumn{4}{|c|}{ the evaluation set $V$} \\
\cline { 2 - 5 } & $\begin{array}{l}v_{1} \text { (very good) } \\
\text { good) }\end{array}$ & $v_{2}$ (good) & \multicolumn{1}{c|}{$v_{3}$ (not so good) } & $v_{4}$ (not \\
\hline$x_{1}$ (GDP) & $80\left(c_{11}\right)$ & $5\left(c_{12}\right)$ & $5\left(c_{13}\right)$ & $10\left(c_{14}\right)$ \\
\hline $\begin{array}{l}x_{2} \text { (urbanization } \\
\text { rate) }\end{array}$ & $85\left(c_{21}\right)$ & $5\left(c_{22}\right)$ & $5\left(c_{23}\right)$ & $5\left(c_{24}\right)$ \\
\hline $\begin{array}{l}x_{3} \text { ( industrialization } \\
\text { rate) }\end{array}$ & $60\left(c_{31}\right)$ & $20\left(c_{32}\right)$ & $10\left(c_{33}\right)$ & $10\left(c_{34}\right)$ \\
\hline $\begin{array}{l}x_{4} \text { ( investment } \\
\text { enviroment) }\end{array}$ & $80\left(c_{41}\right)$ & $10\left(c_{42}\right)$ & $5\left(c_{43}\right)$ & $5\left(c_{44}\right)$ \\
\hline
\end{tabular}

where $c_{i j}(i=1,2,3,4 ; j=1,2,3,4)$ denotes the votes of which the $i$ th factor $x_{i}(i=1,2,3,4)$ is in favor of the $j$ th evaluation $v_{j}(j=1,2,3,4)$ in Table 1 .

If we let $r_{i j}=\frac{c_{i j}}{\sum_{j=1}^{4} c_{i j}}(i=1,2,3,4)$, where $\sum_{j=1}^{4} c_{i j}=100$ in table 1 , then 


$$
R=\left(\begin{array}{cccc}
0.8 & 0.05 & 0.05 & 0.1 \\
0.85 & 0.05 & 0.05 & 0.05 \\
0.6 & 0.2 & 0.1 & 0.1 \\
0.8 & 0.1 & 0.05 & 0.05
\end{array}\right)
$$

(iv) determine the weight of each factor. $\underline{A}=\left(a_{1}, a_{2}, a_{3}, a_{4}\right) \in F(U)$. Here are the weights of each factor determined by two economic experts respectively:

$$
\underline{A}_{1}=(0.45,0.1,0.25,0.2) ; \quad \underline{A}_{2}=(0.3,0.2,0.3,0.2) \text {. }
$$

(v) comprehensive evaluation. According to the fuzzy comprehensive evaluation, we give the fuzzy transformation $\underline{B}=\underline{A} \circ R$. Putting the above data into the fuzzy transformation, it is concluded that the two experts evaluate the overall conditions of Nanchang as follows:

$$
\underline{B}_{1}=\underline{A}_{1} \circ R=(0.45,0.2,0.1,0.1) ; \quad \underline{B}_{2}=\underline{A}_{2} \circ R=(0.3,0.2,0.1,0.1) .
$$

From the above evaluation results, the first expert analysis that the membership degree of the overall conditions of Nanchang high-tech zone belonging to "very good", "good", "not so good" and "not good", are 0.45, 0.2, 0.1 and 0.1, respectively; the second expert analysis that the membership degree of overall conditions of Nanchang high-tech zone belonging to "very good" , "good", "not so good" and "not good", are 0.3, 0.2, 0.1 and 0.1, respectively. Therefore, we say that overall conditions of Nanchang high-tech zone are "very good" by using the principle of maximum membership. Furthermore, we concluded that the present overall conditions of the city of Nanchang is good by using the fuzzy mathematics comprehensive evaluation approach.

\section{Conclusions}

Seen from the above example, a fuzzy mathematics comprehensive evaluation method provides us a scientific method which can make more complex, unclear multi-factor problem into a digital basis and a clear conclusion thing. It provides a reliable basis for decision makers in the related fields. In this paper, we have not done in-depth discussions about the weight of each index. In order to make the evaluation result to be more perfect, those interested in comprehensive evaluation can use the assessment results of normal inspection to determine the weight allocation is reasonable or not.

\section{Acknowledgements}

This work is supported by the National Science Foundation( China ) ( No. 11261018 ), the Social Science Foundation of the Education Department of Jiangxi Province (No. ZGW1204), the Natural Science Foundation of Jiangxi Province ( No. 20122BAB201018 ), the Science Foundation of the Education Department of Jiangxi Province (No. GJJ14381) .

\section{References}

[1] L. Zadeh: Inform. Control Vol . 8(1965) , p.338.

[2] N. Kuroki: Comment. Math. Univ. St. Paul Vol. 28 ( 1979), p. 17

[3] P. Das: Fuzzy Sets and Systems Vol. 105(1999), p.171.

[4] X. Y. Xie: Inform. Sci. Vol. 178(2008), p.4357.

[5] Chunhua Li: International Mathematical Forum Vol. 9( 2014), p.367.

[6] MORDESON J N, MALIK D S and KUROKI N: Fuzzy semigroups (Springer-verlag Berlin Heidelberg New York, 2003).

[7] XIE J J and LIU C P: Fuzzy mathematics method and its application (Huazhong university of science and technology press Wuhan, 2005) (In Chinese). 